\title{
Assessment of Melanoma-Initiating Cell Markers and Conventional Parameters in Sentinel Lymph Nodes of Malignant Melanoma
}

\author{
Norihiro Suzuki ${ }^{a}$, Minoru Takata ${ }^{a}$, Yoshinori Shirafuji ${ }^{a}$, Masaki Otsuka ${ }^{a}$, \\ Osamu Yamasaki ${ }^{a}$, Kenji Asagoe ${ }^{b}$, Naohito Hatta ${ }^{c}$, and Keiji Iwatsuki ${ }^{a *}$ \\ ${ }^{a}$ Department of Dermatology, Okayama University Graduate School of Medicine, Dentistry and Pharmaceutical Sciences, \\ Okayama 700-8558, Japan, ${ }^{b}$ Department of Dermatology, Okayama Medical Center, Okayama 701-1192, Japan, and \\ 'Department of Dermatology, Toyama Prefectural Central Hospital, Toyama 930-0975, Japan
}

\begin{abstract}
Sentinel lymph node (SLN) biopsies have widely been used for the detection of occult LN metastasis of malignant melanoma (MM). In addition to conventional biomarkers, we assessed the diagnostic and prognostic significance of melanoma-initiating cell (MIC) markers in SLNs of MM. We examined the expressions of gp100, MART-1 and tyrosinase mRNA for routine diagnosis and those of ABCB5, CD133, nestin, KDM5B, NGFR and RANK mRNA as MIC markers. The presence of micrometastasis was confirmed immunohistochemically using antibodies to S-100, HMB-45, MART-1, and tyrosinase. Discordance between immunohistochemical and molecular data was observed in 14 of $70(20.0 \%)$ patients, among whom five $\mathbf{( 7 . 1 \% )}$ ) were positive for only molecular markers; two of these five patients tested positive for micrometastasis by repeated immunohistochemical stainings. The quantitative expression levels of gp100, MART-1, and tyrosinase mRNA were significantly higher in the metastatic LNs; the cut-off values remain to be elucidated. ABCB5 mRNA expression was detected more frequently in the metastatic SLNs $(\phi<0.05)$ and in the group of patients with recurrence. To make a definite diagnosis of metastasis, we still need a combination of immunohistochemical and molecular probes. ABCB5 might be a suitable molecular marker for the detection of melanoma-initiating cells in SLNs.
\end{abstract}

Key words: melanoma, cancer-initiating cell, sentinel lymph node, ABCB5

$\mathbf{T}$ he authors of a large-scale clinical study concluded that the sentinel lymph node (SLN) biopsy-based management of malignant melanoma (MM) prolonged the disease-free survival and melanomaspecific survival of a group of patients with nodal metastases of intermediate-thickness melanomas, defined as 1.20 to $3.50 \mathrm{~mm}$ [1]. Standard SLN biopsies were

Received July 11, 2014; accepted September 10, 2014.

*Corresponding author. Phone:+81-86-235-7282; Fax:+81-86-235-7283

E-mail:keijiwa@cc.okayama-u.ac.jp (K. Iwatsuki) performed using 3 steps: (1) lymphoscintigraphy, followed by (2) intraoperative injection of vital dye around the primary tumor, and (3) confirmation of the radioactivity of the LNs by gamma probe [2]. Although the accurate detection of metastasis in SLNs is essential for the management of MM patients, both false-positive and false-negative results may occur even though sensitive immunohistochemical methods

Conflict of Interest Disclosures: No potential conflict of interest relevant to this article was reported. 
using specific antibodies to MART-1/Melan-A, HMB45, S-100 protein and Tyrosinase are used [3]. Although quantitative RT-PCR methods using a primer set specific for gp100, MART-1, and tyrosinase facilitate the detection of occult metastases in SLNs, it is still unknown whether such micrometastases detected only by PCR have prognostic significance $[4]$.

Recent studies have demonstrated the presence of cancer-initiating cells (CICs) with stem cell-like characteristics [5-8]. In melanoma, similar CIC populations designated as melanoma-initiating cells (MICs) have been identified. These MICs have been reported to express specific cell surface markers including ABCB5, CD133, nestin, KDM5B (JARID1B), NGFR (CD271) and RANK [9-14]. In addition to the conventional cellular and molecular markers used for evaluating SLN biopsies, we have examined the expression of some MIC-related markers in SLNs, and assessed the possibility of using these MIC markers as prognostic indicators, as MICs are thought to play a critical role in melanoma progression $[9,12]$.

\section{Materials and Methods}

Patients. The study was approved by the Ethical Committee of the Okayama University School of Medicine (no. 968) and carried out according to the guidelines set by the Institutional Review Board.

The study included a total of 70 patients who underwent an SLN biopsy for primary cutaneous melanoma between 1997 and 2010 at Okayama University Hospital (Okayama, Japan). The clinical and pathologic characteristics of the 70 patients are listed in Table 1 . There were 33 men and 37 women, and the median age was 60.6 years. More than onehalf of the primary melanomas were located on an extremity, followed by the trunk and the head and neck. The median and mean tumor thicknesses were $2.70 \mathrm{~mm}$ and $2.41 \mathrm{~mm}$, respectively (range $0-13 \mathrm{~mm}$ ). Ulceration of the primary tumor was present in $45.7 \%$ of the cases.

Tissues. A total of 141 SLNs were identified and excised from 70 melanoma patients. Fresh frozen tissues of 14 primary melanoma tumors, seven normal skin samples, and 18 non-melanoma lymph nodes were used for molecular analyses.

Pathological and immunohistochemical stud-
Table 1 Patient clinicopathological data

\begin{tabular}{|c|c|c|c|}
\hline \multirow[t]{2}{*}{ Mean age (years) } & & \multicolumn{2}{|c|}{60.6} \\
\hline & & $n$ & $\%$ \\
\hline \multirow[t]{2}{*}{ Sex } & Male & 33 & 47.1 \\
\hline & Female & 37 & 52.9 \\
\hline \multirow[t]{3}{*}{ Primary Site } & Head and neck & 13 & 18.6 \\
\hline & Trunk & 16 & 22.9 \\
\hline & Extremities & 41 & 58.6 \\
\hline \multirow[t]{8}{*}{ Breslow thickness } & $\leqq 1.00$ & 10 & 14.3 \\
\hline & $1.01-2.00$ & 13 & 18.6 \\
\hline & $2.01-4.00$ & 26 & 37.1 \\
\hline & $>4.00$ & 20 & 28.6 \\
\hline & Unknown & 1 & 1.4 \\
\hline & Mean & 2.41 & \\
\hline & Median & 2.7 & \\
\hline & Range & $0-13$ & \\
\hline \multirow[t]{3}{*}{ Ulceration } & Present & 32 & 45.7 \\
\hline & Absent & 38 & 54.3 \\
\hline & Unknown & 0 & 0.0 \\
\hline total $N$ & & 70 & \\
\hline
\end{tabular}

ies for SLNs. A total of 141 SLNs from 70 melanoma patients were subjected to histological and molecular genetic analyses. To detect SLN metastasis, we used a central slice of each SLN for the histological diagnosis. Each SLN was stained with hematoxylin-eosin (HE). To confirm micrometastases, we stained all of the sections with antibodies to S100 (DAKO, Glostrup, Denmark), MART-1 (clone M2-7C10, COVANCE, Dedham, MA, USA), tyrosinase (clone T311, Leica Biosystems, Nussloch, Germany) and HMB-45 (clone HMB45, DAKO) antibodies. The secondary antibody was Histofine ${ }^{\circledR}$ Simple Stain MAX PO (MULTI) (Nichirei Biosciences, Tokyo, Japan).

Conventional RT-PCR analysis. The peripheral portions of the SLNs were snap-frozen and used for molecular genetic analyses. Eighteen histologically proven normal lymph nodes (nLNs) from patients with non-melanoma disease were used as a control. We extracted total RNA from the LN samples using the RNeasy Mini Kit (Qiagen, Hilden, Germany), and the total RNA was reverse-transcribed using the SuperScript First Strand Synthesis System (Invitrogen, Carlsbad, CA, USA). The primers used for the detection of target genes (gp100, MART-1, 
tyrosinase, GAPDH) were as follows: gp100,

5'-GCTTGGTGTCTCAAGGCAACT-3' and 5'-CTCCAGGTAAGTATGAGTGAC-3'; MART-1, 5'CACGGCCACTCTTACACCAC-3' and 5'-GGAGCATTGGGAACCACAGG-3'; and tyrosinase, 5'-TTGGCAGATTGTCTGTAGCC-3' and 5'AGGCATTGTGCATGCTGCTT-3'.

PCR was performed in the following manner: one cycle of denaturation at $94^{\circ} \mathrm{C}$ for $2 \mathrm{~min}$ as the first step, followed by 35 successive cycles of PCR comprising $94^{\circ} \mathrm{C}$ for $1 \mathrm{~min}, 53^{\circ} \mathrm{C}$ for $1 \mathrm{~min}$, and $72^{\circ} \mathrm{C}$ for $1 \mathrm{~min}$. The PCR products were analyzed by electrophoresis using a $1 \%$ agarose gel. After electrophoresis, the gels were photographed using ultraviolet transillumination.

Quantitative reverse transcription (qRT)PCR analysis. TaqMan Gene Expression Assays (Applied Biosystems ABI, Foster City, CA, USA) were used to analyze the expression levels of human GP100 (assay ID: Hs00202752_m1), human MART-1 (assay ID: Hs00192503_m1), and human tyrosinase (assay ID: Hs00166289_m1) in 90 SLNs from 48 patients. In 83 SLNs from 43 patients, we also performed quantitative RT-PCR analyses for putative MIC markers, including human ABCB5 (assay ID: Hs00846307_s1), human CD133 (assay ID: Hs00202752_m1), human nestin (assay ID: Hs00192503_m1), human JARID1B (assay ID: Hs00846307_s1), human NGFR (CD271) (assay ID: Hs00166289_m1) and human RANK (assay ID: Hs00166289_m1) following the manufacturer's instructions (User bulletin \#2 by Applied Biosystems).

GAPDH mRNA was detected by the probe VICCATCCATGACAACTTTGGTAMGB and using the primers 50-CTTAGCACCCCTGGCCAAG-30 and 50-TGGTCAT GAGTCCTTCCACG-3, and it was used as an internal control to validate the RNA for each sample. We also performed quantitative reverse transcription (qRT)-PCR analyses for MIC markers in 14 primary melanoma tissues, seven normal skin samples, and 18 non-melanoma lymph nodes. Each mRNA expression was calculated as the expression relative to GAPDH mRNA. Each assay was performed in duplicate, and the mean threshold cycle $(\mathrm{Ct})$ was used for the analysis.

\section{Results}

\section{Effectiveness of a combination study with immunohistochemical and molecular markers.}

The removed SNLs were first screened by both an immunohistochemical study using antibodies to S-100, HMB-45, MART-1 and tyrosinase (Fig. 1) and RT-PCR amplification using primer sets specific for gp100, MART-1 and tyrosinase cDNA (Fig. 2). The SLNs were defined as metastasis-positive when a group of atypical cells was positive for one or more of the immunohistochemical markers, even if the samples were negative for the molecular markers. If a positive result was obtained by molecular markers alone, but a negative result was obtained for immunohistochemical markers, the samples were re-examined using immunohistochemistry (IHC).

Discordance between the immunohistochemical and molecular data indicating metastasis was observed in 14 of the $70(20 \%)$ patients, among whom five $(7.1 \%)$ patients were positive for only molecular markers and nine patients $(12.9 \%)$ were positive for only immunohistochemical markers (Table 2). Two of the five (40\%) patients who were positive only by RT-PCR were revealed to have micrometastases by repeated immunohistochemical staining. In the remaining three patients metastases could still not be detected after re-evaluation by immunohistochemistry. We have observed no recurrence in the three patients who were positive only by RT-PCR while recurrence and death occurred in nine patients who were positive only by immunohistochemical markers.

Quantitative analysis of MM-associated molecules in the SLNs. The quantitative expressions of gp100, MART-1, and tyrosinase mRNA were significantly higher in the metastatic SLNs than in the non-metastatic SLNs (Fig. 3).

The metastatic SLNs were confirmed by HE and IHC stains. However, it was difficult to set the cut-off values because of the wide range of values. In the 15 tumor-positive sentinel lymph nodes that were confirmed by $\mathrm{HE}$ and IHC, we evaluated the expression levels of gp100, MART-1, and tyrosinase mRNA by determining the distance of tumor cell invasion from the capsules, based on the following definitions: S1, $\leq 0.3 \mathrm{~mm}$; $\mathrm{S} 2,>0.3 \leq 1.0$; and $\mathrm{S} 3,>1.0 \mathrm{~mm}[15]$. The expression levels tended to increase according to the $\mathrm{S}$ classification, but the difference was not sig- 


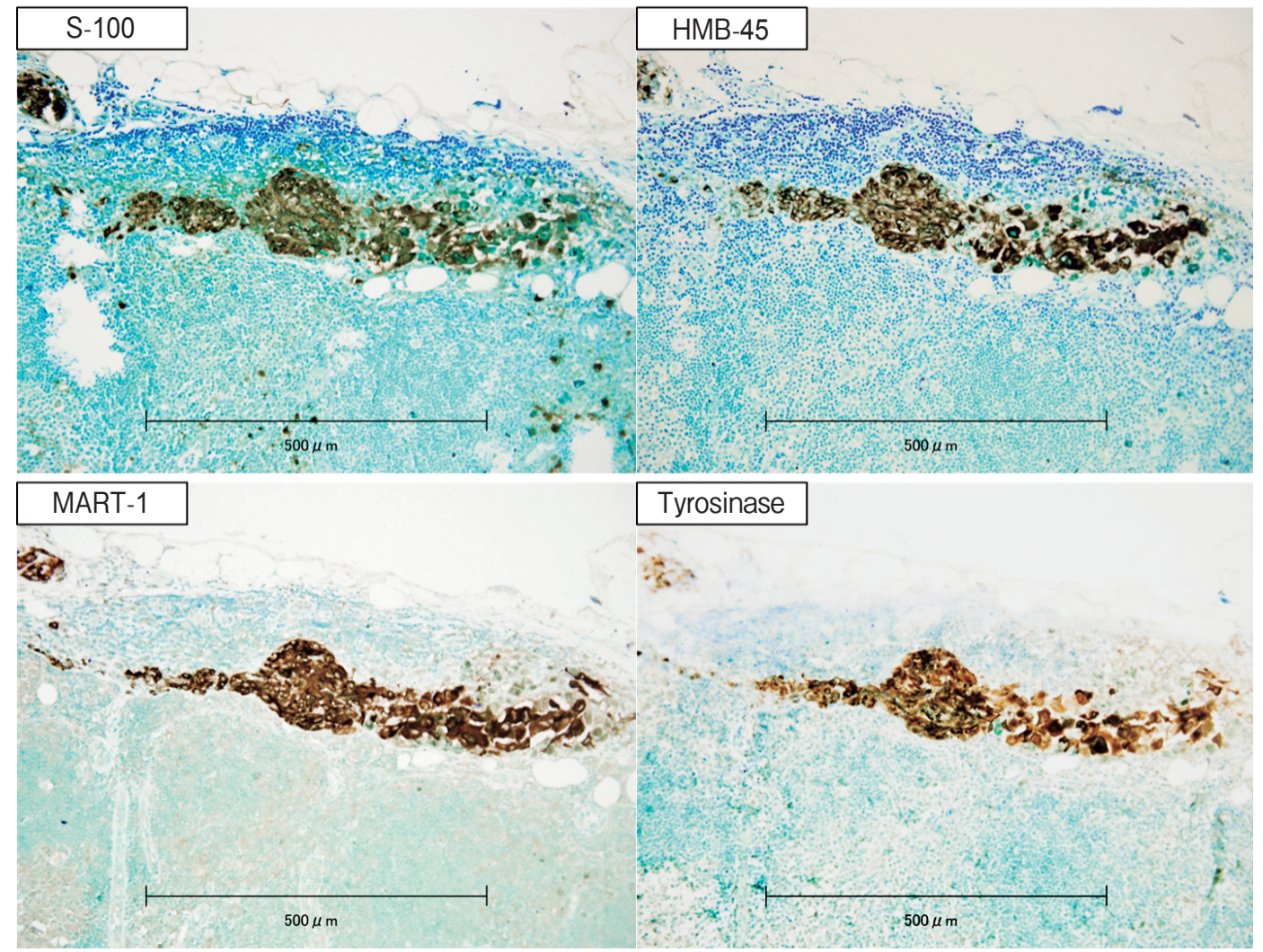

Fig. 1 Immunohistochemical stains of SLNs of malignant melanoma. To confirm micrometastases, we stained all of the sections with antibodies to S100, MART-1, HMB-45 and tyrosinase antibodies.

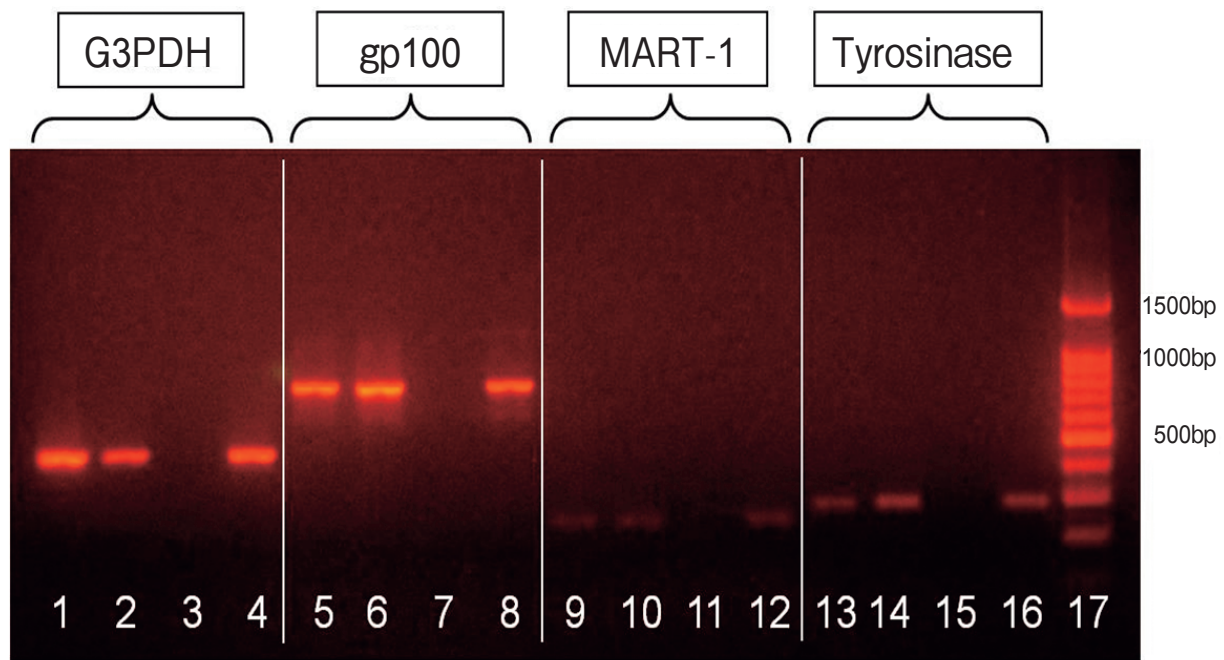

$1,5,9,13$; sentinel lymph node

$2,6,10,14$; primary lesion of melanoma

$3,7,11,15$; negative control

$4,8,12,16$; positive control

Fig. 2 RT-PCR assays in SLNs of malignant melanoma. The peripheral portions of the sentinel lymph nodes (SLNs) were snap-frozen and used for molecular genetic analyses. RT-PCR amplification using primer sets specific for gp100, MART-1 and tyrosinase cDNA. GAPDH was used as a housekeeping gene. 
Table 2 Histological and molecular analysis in SLNs of melanoma

\begin{tabular}{|c|c|c|c|}
\hline \multirow{2}{*}{$\begin{array}{c}\text { Histological } \\
\text { analysis } \\
(\mathrm{HE} / \mathrm{IHC})\end{array}$} & \multirow{2}{*}{$\begin{array}{l}\text { Molecular } \\
\text { analysis } \\
\text { (RT-PCR) }\end{array}$} & \multicolumn{2}{|c|}{ Results } \\
\hline & & Patients (\%) & Lymph nodes \\
\hline- & - & $38 / 70(54.3 \%)$ & $94 / 141$ \\
\hline- & + & $\begin{array}{r}5 / 70(7.1 \%) \\
* \rightarrow 3 / 70(4.3 \%)\end{array}$ & $\begin{array}{r}11 / 141 \\
9 / 141\end{array}$ \\
\hline+ & - & $9 / 70(12.9 \%)$ & $11 / 141$ \\
\hline+ & + & $\begin{array}{r}18 / 70(25.7 \%) \\
* \rightarrow 20 / 70(28.6 \%)\end{array}$ & $\begin{array}{l}25 / 141 \\
27 / 141\end{array}$ \\
\hline & & $70 / 70$ & $141 / 141$ \\
\hline
\end{tabular}

+ (histological analysis); tumor cells confirmed by $\mathrm{HE}$ and or at least one marker stained by immunohistochemical study.

+ (molecular analysis); at least one marker expression of mRNA confirmed by RT-PCR.

* Two of the five patients who were positive only by RT-PCR were revealed to have micrometastases by repeated immunohistochemical staining. nificant (Fig. 4).

The expression of MIC markers in the SLNs. Among the six MIC markers including ABCB5, CD133, nestin, KDM5B, NGFR and RANK mRNA, the ABCB5 mRNA was specifically detected in the primary lesions of MM that metastasized to the SLNs, but not in the cases without metastasis or normal skin (Fig. 5), although a significant difference was not observed. The expression of ABCB5 mRNA in primary lesions was detected in four of the eight $(50 \%)$ patients with metastasis to SLNs, and in three of the six $(50 \%)$ patients without metastasis. One of the former four patients had recurrence in the mediastinum LNs and died, while the latter three patients had no recurrence. The KDM5B mRNA expression levels were significantly lower in the primary lesions compared to the normal skin samples $(p<0.05)$.

In the SLNs, the expression levels of ABCB5 mRNA were higher in the metastasis-positive LNs compared to the metastasis-negative and normal LNs $(p<0.05)$. Up-regulation was not observed in the expression levels of CD133, nestin, KDM5B, NGFR or RANK mRNA in any group (Fig. 6). These results indicated that $\mathrm{ABCB} 5$ might be a candidate marker for
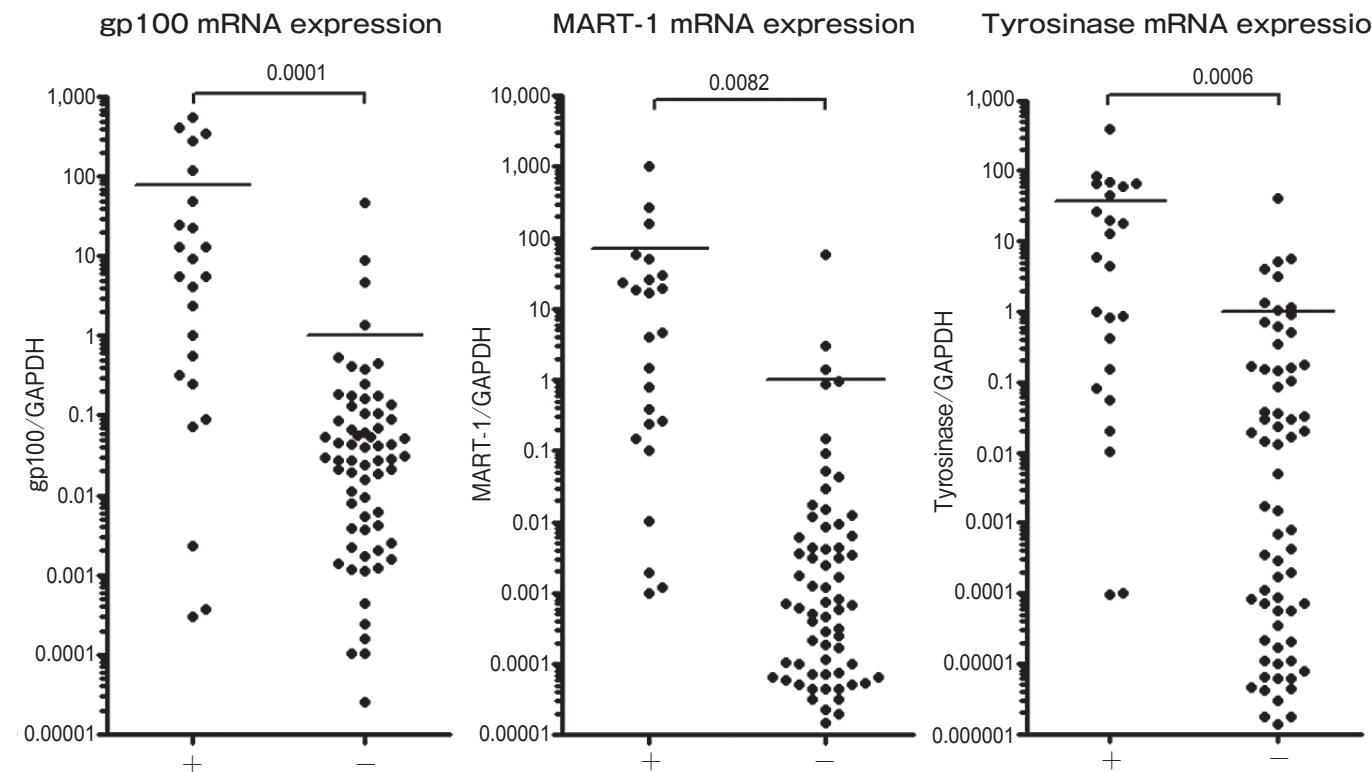

+; a tumor positive SLN confirmed by HE and Immunohisitochemical (IHC) stains $(n=24)$

-; a tumor negative SLN confirmed by HE and IHC stains $(n=66)$

Fig. 3 The quantitative expression of gp100, MART-1, and tyrosinase mRNA in SLNs. The quantitative expressions of gp100, MART-1, and tyrosinase mRNA were significantly higher in the metastatic SLNs than in the non-metastatic SLNs. 

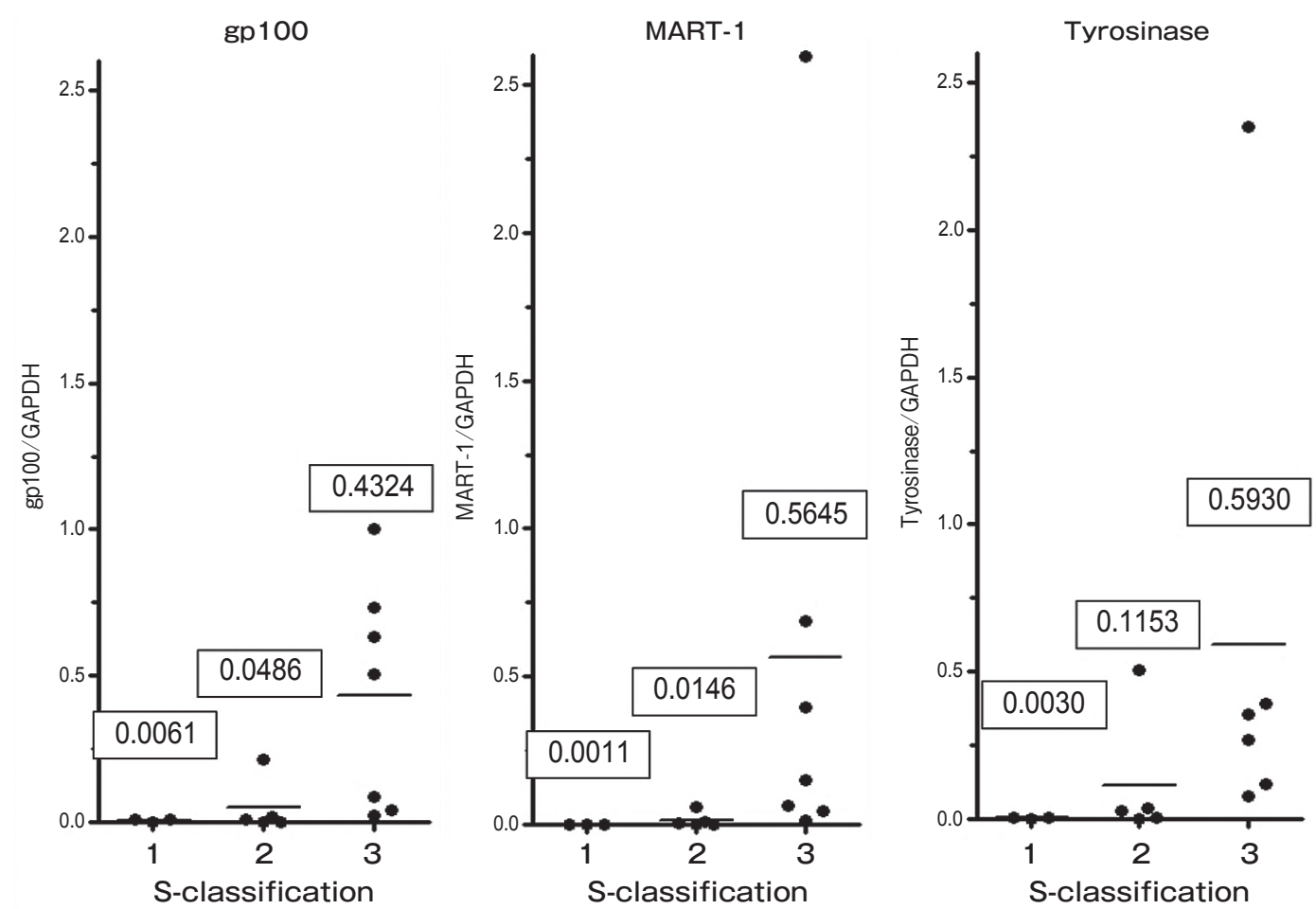

S-classificatin was deifined by the distance of tumor cell invasion from the capsules. $\mathrm{S} 1 ; 0.3 \mathrm{~mm}$ or less $(n=3)$, S2; more than 0.3 until $1.0 \mathrm{~mm}$ or less $(n=5)$, and $S 3$; more than $1.0 \mathrm{~mm}(n=7)$.

Fig. 4 The quantitative expression of gp100, MART-1, and tyrosinase mRNA in S-classifications of tumor-positive SLNs. In the 15 tumor-positive sentinel lymph nodes that were confirmed by HE and IHC, we evaluated the expression levels of gp100, MART-1, and tyrosinase mRNA by determining the distance of tumor cell invasion from the capsules. The expression levels tended to increase according to the $\mathrm{S}$ classification, but the differences were not significant.

the detection of MICs in SLNs as well as primary lesions, and for the prediction of recurrence.

Prediction of the recurrence of MM by conventional molecular markers and $A B C B 5 . \quad$ Of the 41 patients who underwent SLN biopsy, nine experienced recurrence. ABCB5 mRNA was detected in seven of the $32(21.9 \%)$ patients without recurrence, and in four of the nine $(44.4 \%)$ patients with recurrence. The expression of ABCB5 mRNA might be found more frequently in MM patients with recurrence.

In contrast, eight of the nine patients $(88.9 \%)$ in the recurrence group expressed all three of the molecular markers, MART-1, tyrosinase, and gp100 mRNA, while the non-recurrence group showed a low three-way positivity, in only seven of the 32 (21.9\%). Thus, the detection of all of three molecular markers MART-1, tyrosinase, and gp100 mRNA may predict the recurrence more accurately than the expression of
ABCB5 mRNA alone.

A total of 15 patients were positive for all three conventional RT-PCR markers, and eight of them experienced relapse. Four of the eight patients with relapse were also positive for ABCB5, while six of the seven patients without relapse were negative for ABCB5 ( $p=0.14$, by chi square test). Thus, ABCB5 expression in SNLs may help to further stratify the group of patients who are positive for all three conventional markers, MART-1, tyrosinase, and gp100 (Table 3).

\section{Discussion}

In addition to conventional immunohistochemical markers, we assessed the usefulness of the molecular markers gp100, MART-1 and tyrosinase mRNA. Five of 70 patients $(7.1 \%)$ were negative in the immunohistochemical study but positive for one or more of the 

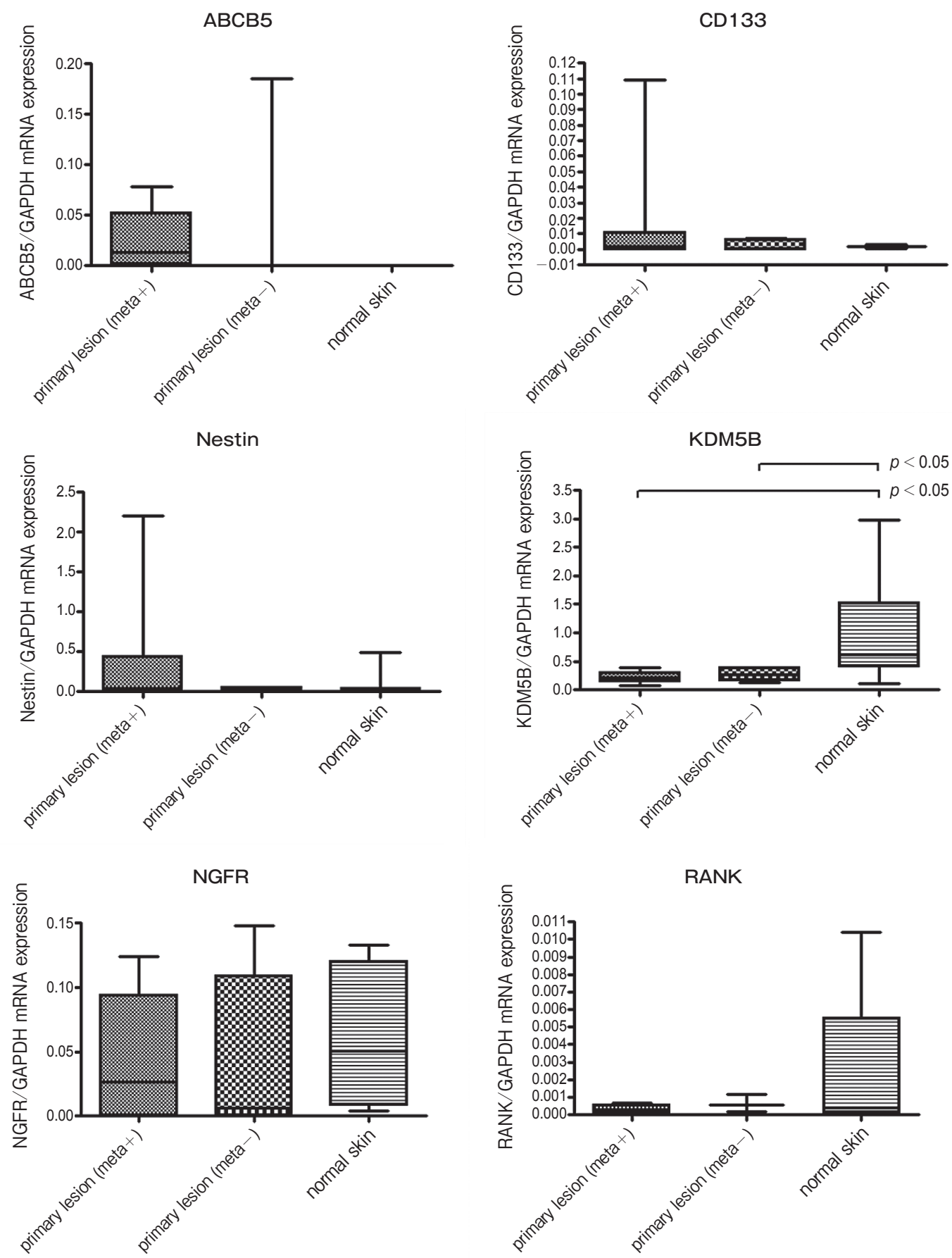

Fig. 5 The expression of MIC markers in primary lesions of MM and normal skin. Primary lesions with SLN metastasis confirmed by HE and IHC $(n=8)$. Primary lesions without SLN metastasis confirmed by HE and IHC $(n=6)$. Normal skin samples $(n=7)$. Among the six MIC markers including ABCB5, CD133, Nestin, CD271, JARID1B and RANK mRNA, the ABCB5 mRNA was specifically detected in the primary lesions of MM that metastasized to the SLNs, but not in the cases without metastasis or normal skin. The KDM5B mRNA expression levels were significantly lower in the primary lesions compared to the normal skin samples $(p<0.05)$. 
ABCB5
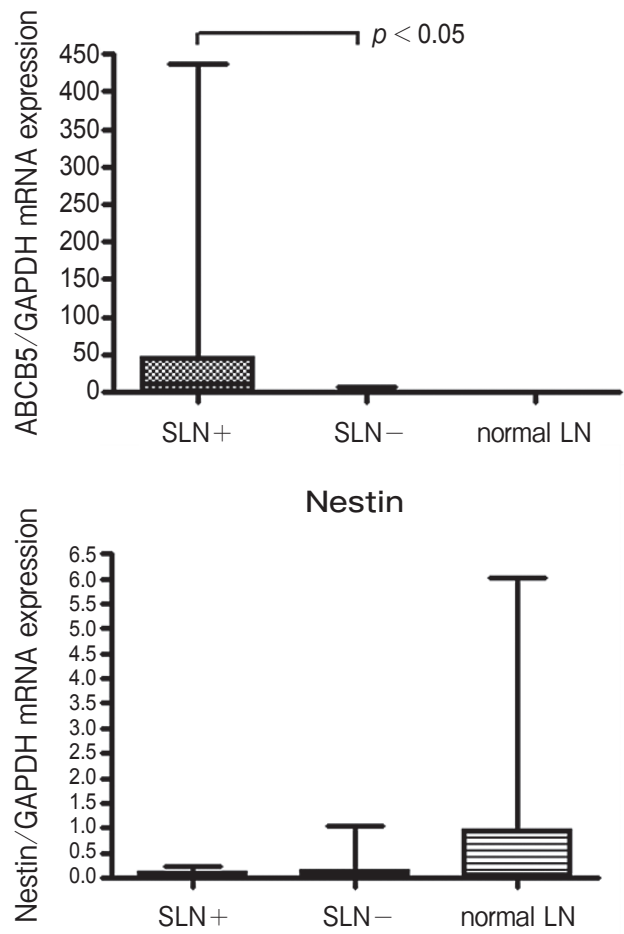

NGFR

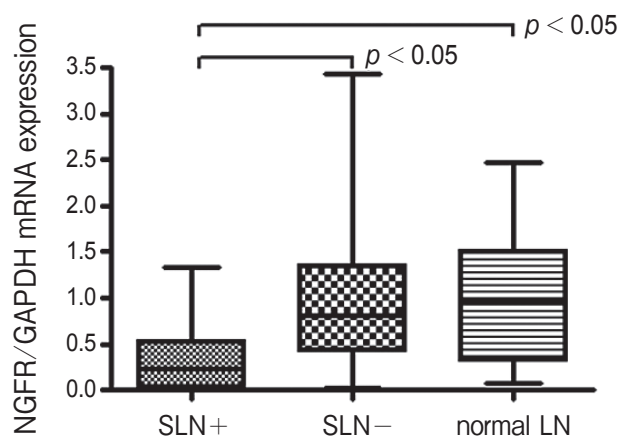

CD133

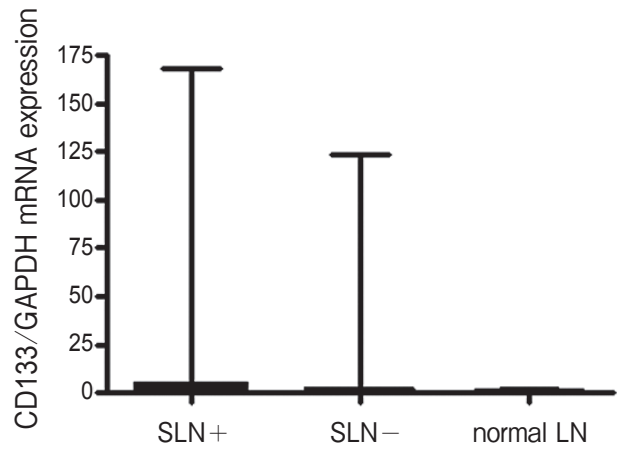

KDM5B
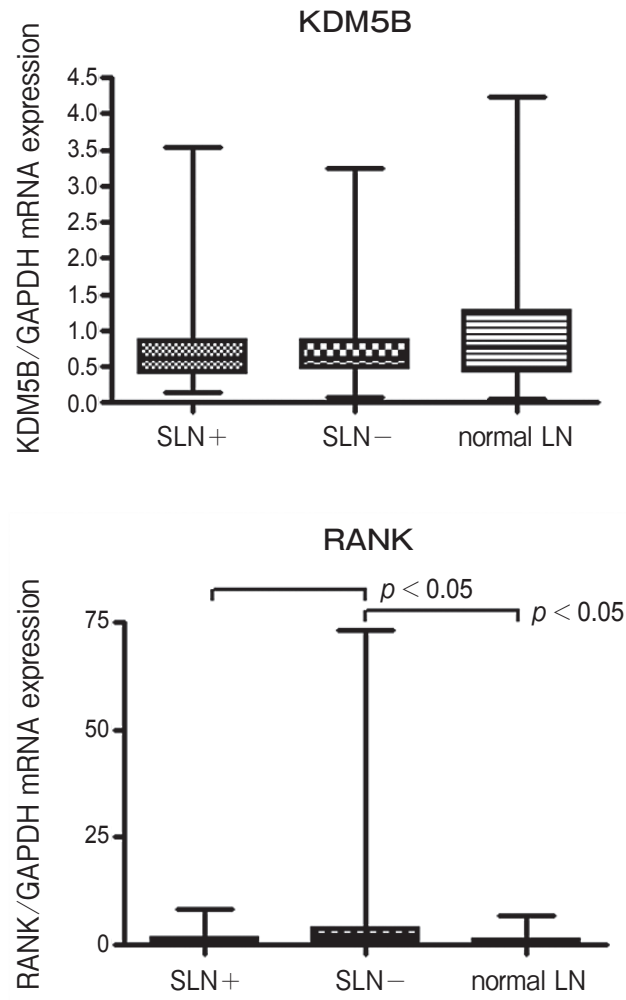

Fig. 6 The expression of MIC markers in melanoma SLNs and normal LNs. SLN+, a tumor-positive SLN confirmed by HE and IHC stains $(n=23)$. SLN - , a tumor-negative SLN confirmed by HE and IHC stains $(n=60)$. Normal LN, lymph nodes from patients with non-melanoma disease $(n=18)$. In the SLNs, the expression levels of ABCB5 mRNA were higher in the metastasis-positive LNs than in the metastasis-negative and normal LNs $(p<0.05)$. No clear differences were observed in the expression levels of CD133, nestin, KDM5B and NGFR mRNA in each group.

molecular markers. In our confirmation study, the further sectioned specimens turned out to be positive in two of these five patients by immunohistochemical examination. The false-negative results were thus corrected by the detection of molecular markers.

In contrast, nine of the 70 patients $(12.9 \%)$ who were positive in the immunohistochemical examination showed a negative result in the molecular analysis. Since the RT-PCR assay is thought to be far more sensitive than the immunohistochemical method used here, it might be critical that the tissue specimens are sectioned from the proper portion of the SLNs containing metastatic cells.

It is widely agreed that an H\&E-based histopatho- 
Table 3 Clinical and molecular features of 41 patients of malignant melanoma

\begin{tabular}{|c|c|c|c|c|c|c|c|c|c|c|c|c|c|c|c|}
\hline \multirow{2}{*}{ Pt No. } & \multirow{2}{*}{ Age } & \multirow{2}{*}{ Sex } & \multirow{2}{*}{ TT } & \multirow{2}{*}{ Ulcer } & \multirow{2}{*}{ Stage } & \multirow{2}{*}{$\mathrm{HE} / \mathrm{IHC}$} & \multirow{2}{*}{ S-class } & \multicolumn{3}{|c|}{ RT-PCR } & \multirow{2}{*}{$\frac{\mathrm{qRT}-\mathrm{PCR}}{A B C B 5}$} & \multirow{2}{*}{ Chemotherapy } & \multirow{2}{*}{ Recurrence } & \multirow{2}{*}{ Death } & \multirow{2}{*}{ OS (months } \\
\hline & & & & & & & & MART-1 & TYR & GP100 & & & & & \\
\hline 1 & 74 & $\mathrm{M}$ & 3.25 & + & II B & - & & - & - & - & - & IFN $\beta$ & & $?$ & $?$ \\
\hline 2 & 46 & $F$ & 6.5 & + & IIIA & + & 2 & + & + & + & - & D-IFN $\beta 2$ course & +(lung meta.) & + & 62 \\
\hline 3 & 63 & $\mathrm{~F}$ & 0.75 & - & IIIA & + & 3 & + & + & + & + & D-IFN $\beta 6$ course & + (mediastinal meta.) & + & 82 \\
\hline 4 & 64 & $\mathrm{M}$ & 5.2 & + & IIIC & + & 3 & + & + & + & + & IFN $\beta$ & + (liver meta.) & + & 69 \\
\hline 5 & 69 & $\mathrm{M}$ & 8.4 & + & IIIA & + & 1 & + & + & + & - & D-IFN $\beta 6$ course & + (liver meta.) & + & 44 \\
\hline 6 & 71 & $\mathrm{M}$ & 2.05 & + & II A & - & & $+?$ & - & $+?$ & - & ND & & - & 96 \\
\hline 7 & 49 & $\mathrm{~F}$ & 4.2 & - & IIIA & + & 2 & + & + & + & - & D-IFN $\beta 6$ course & & - & 95 \\
\hline 8 & 78 & $\mathrm{M}$ & 3.9 & + & IIIC & + & 2 & + & - & - & + & D-IFN $\beta 6$ course & & - & 92 \\
\hline 9 & 66 & $\mathrm{M}$ & 2.2 & - & II A & - & & - & - & - & - & D-IFN $\beta 3$ course & & - & 87 \\
\hline 10 & 84 & $F$ & 5.9 & + & II C & - & & - & - & - & - & ND & & - & 84 \\
\hline 11 & 68 & $\mathrm{M}$ & 3.3 & - & IIIA & + & 3 & + & + & + & - & D-IFN $\beta 6$ course & & - & 82 \\
\hline 12 & 73 & $\mathrm{~F}$ & $>4.0$ & - & II B & - & & - & - & - & + & D-IFN $\beta 3$ course & & - & 79 \\
\hline 13 & 69 & $\mathrm{M}$ & 3.5 & - & IIIB & + & 3 & - & - & - & - & ND & & $?$ & $?$ \\
\hline 14 & 57 & $\mathrm{M}$ & 1.7 & + & II A & - & & - & - & - & - & D-IFN $\beta 3$ course & & - & 74 \\
\hline 15 & 58 & $\mathrm{~F}$ & 1.5 & + & II A & - & & + & + & + & - & D-IFN $\beta 3$ course & & - & 70 \\
\hline 16 & 27 & $\mathrm{~F}$ & 2.1 & + & II B & - & & - & - & - & - & D-IFN $\beta 3$ course & & - & 70 \\
\hline 17 & 43 & $\mathrm{~F}$ & 0.8 & - & I A & - & & - & - & - & - & ND & & - & 69 \\
\hline 18 & 75 & $\mathrm{~F}$ & 2.9 & + & II B & - & & - & - & - & - & D-IFN $\beta 3$ course & & - & 68 \\
\hline 19 & 78 & $\mathrm{M}$ & 7.2 & + & IIIB & + & 3 & + & + & + & + & D-IFN $\beta 6$ course & +(lung meta.) & + & 37 \\
\hline 20 & 78 & M & 7.2 & + & IIIB & + & 1 & + & + & + & - & D-IFN $\beta 2$ course & + (mediastinal LN meta.) & + & 27 \\
\hline 21 & 64 & $\mathrm{M}$ & 1.3 & + & IIIB & + & 3 & - & - & $+?$ & + & D-IFN $\beta 5$ course & & - & 65 \\
\hline 22 & 84 & $\mathrm{~F}$ & 1.6 & - & IIIA & + & 2 & + & + & $+?$ & + & ND & & - & 64 \\
\hline 23 & 76 & $\mathrm{~F}$ & in situ & - & 0 & - & & - & - & $+?$ & + & ND & & - & 62 \\
\hline 24 & 32 & $\mathrm{M}$ & 13 & + & II C & - & & - & - & - & - & D-IFN $\beta 4$ course & & - & 60 \\
\hline 25 & 78 & $\mathrm{M}$ & 1.8 & + & II A & - & & - & - & - & - & D-IFN $\beta 2$ course & & - & 58 \\
\hline 26 & 91 & $\mathrm{~F}$ & 5.6 & + & IIIC & + & 3 & + & + & + & + & ND & & $*+$ & 46 \\
\hline 27 & 74 & $\mathrm{~F}$ & 2.8 & + & II B & - & & + & + & + & - & IFN $\beta$ & & - & 57 \\
\hline 28 & 51 & $\mathrm{~F}$ & 1.2 & + & II A & - & & - & - & - & - & D-IFN $\beta 2$ course & & - & 54 \\
\hline 29 & 66 & $\mathrm{~F}$ & 10 & + & IIIB & + & 3 & - & - & + & - & D-IFN $\beta 4$ course & +(lung, liver, brain meta.) & + & 8 \\
\hline 30 & 75 & $\mathrm{M}$ & 7.2 & - & IIIB & - & & - & - & - & - & IFN $\beta$ & & - & 51 \\
\hline 31 & 75 & $\mathrm{~F}$ & 0.9 & - & IIIA & + & & - & - & - & + & D-IFN $\beta 5$ course & & - & 51 \\
\hline 32 & 21 & $\mathrm{~F}$ & 5 & + & II C & - & & - & - & - & - & D-IFN $\beta 5$ course & & - & 50 \\
\hline 33 & 22 & $\mathrm{M}$ & 0.9 & - & I A & - & & - & - & - & - & ND & & - & 49 \\
\hline 34 & 22 & M & 2.7 & - & II B & - & & - & - & - & - & D-IFN $\beta 3$ course & & - & 49 \\
\hline 35 & 81 & $\mathrm{~F}$ & in situ & - & 0 & - & & - & - & - & - & ND & & - & 48 \\
\hline 36 & 57 & $\mathrm{~F}$ & 3.2 & - & II A & - & & - & - & - & - & D-IFN $\beta 3$ course & & - & 47 \\
\hline 37 & 71 & $\mathrm{~F}$ & 4.2 & + & IIIB & + & 3 & - & - & - & - & D-IFN $\beta 5$ course & & - & 43 \\
\hline 38 & 61 & $\mathrm{M}$ & 1.1 & - & IIIA & + & 2 & + & + & + & - & D-IFN $\beta 5$ course & & - & 45 \\
\hline 39 & 87 & $\mathrm{~F}$ & 13 & + & IIIC & + & 3 & + & + & + & + & IFN $\beta$ & + (in-transit meta.) & + & 11 \\
\hline 40 & 71 & $\mathrm{M}$ & 3.8 & + & IIIB & + & 2 & + & + & + & - & D-IFN $\beta 6$ course & + (in-transit meta.) & + & 39 \\
\hline 41 & 69 & $F$ & $>4.0$ & + & IIIB & + & 2 & + & + & + & - & IFN $\beta$ & & - & 31 \\
\hline
\end{tabular}

TT, tumor thicness; HE, Hematoxylin and Eosin Staining; IHC, Immonohistochemistry Staing; TYR, tyrosinase; OS, overall survival; IFN $\beta$, interferon $\beta$ local injection; D-IFN $\beta$, DTIC intravenous injection + interferon $\beta$ local injection; ND, not done; $*$, death of other diseases. 
logical analysis alone results in an unacceptably high rate of false-negative results. It was shown that in patients who developed metastases in a regional lymph node basin that was originally diagnosed as negative by a histopathological SLN evaluation, re-evaluation by immunohistochemistry revealed SLN micrometastases in $80 \%$ of the cases [16]. In a retrospective analysis of the 143 patients with initially histopathologically negative sentinel lymph node biopsies, 10 of the 15 cases $(67 \%)$ with nodal progression were found to be false-negative due to a previous erroneous routine reading of $H \& E$ specimens [17]. However, in another study a rate of only $12 \%$ false-negative results was reported [18]. The accurate detection of micrometastases therefore requires the combination of histopathological analysis and a molecular analysis.

Our quantitative RT-PCR assay for the molecular markers demonstrated that the expression levels of gp100, MART-1, and tyrosinase mRNA were significantly higher in the metastatic LNs compared to the non-metastatic LNs $(p<0.05)$ and were related to the distance of invasion from the LN capsule. A quantitative assay is thus useful for comparative studies of metastatic and non-metastatic groups, but it is difficult to show the evidence of metastasis in individual cases.

Of the CIC markers examined in the present study, the expression of ABCB5 mRNA was more specific for the primary melanoma tissue than the other CIC markers, although the positivity rates were rather low.

In the metastatic LNs, ABCB5 mRNA expression was detected significantly more frequently than in the non-metastatic SLNs $(p<0.05)$. A follow-up study revealed that the ABCB5 mRNA-positive cases had a higher frequency of recurrence compared to that of the negative group ( $44.4 \%$ vs. $21.9 \%)$. ABCB5 is a novel human $\mathrm{ABC}$ transporter encoded on chromosome $7 \mathrm{p} 15.3$. Previous studies have shown that ABCB5, like ABCB1, acts as an energy-dependent drug efflux transporter for the fluorescent probe rhodamine- 123 . Moreover, ABCB5 marks CD133+ progenitor cells among human epidermal melanocytes (HEMs), and the transporter is expressed by a subset of G3361 malignant melanoma cells.

In physiological progenitor cells, ABCB5 functions to maintain membrane hyperpolarization, thereby serving as a negative regulator of cell fusion of the expressing progenitor subset and, as a consequence, of culture growth and differentiation. Remarkably, membrane hyperpolarization is associated with the MDR phenotype of certain cancer cells, and although the potential role of $\mathrm{ABCB} 1$ as a determinant of membrane potential was examined recently, this transporter was not found to be responsible in the investigated cancer types [19]. It has been reported that the melanoma chemoresistance mediator ABCB5 could represent a molecular marker defining tumorigenic malignant-melanoma-initiating cells (MMICs), because its expression also characterizes progenitor cell subsets in physiological skin [9].

In conclusion, although ABCB5 is a possible molecular marker for the detection of melanoma-initiating cells in SLNs, its diagnostic value for the prediction of recurrence should be studied carefully. At the present time, in order to make the definite diagnosis of metastasis, a combination of results from immunohistochemical and molecular probes is still required.

Acknowledgments. This work was partly supported by a Grant-inAid for Scientific Research (C) from the Ministry of Education, Culture, Science and Technology (MEXT), Japan (M.T: \#C, 2351619, and M.O: \#C, 23791269)

\section{References}

1. Morton DL, Thompson JF, Cochran AJ, Mozzillo N, Nieweg OE, Roses DF, Hoekstra HJ, Karakousis CP, Puleo CA, Coventry BJ, Kashani-Sabet M, Smithers BM, Paul E, Kraybill WG, McKinnon JG, Wang HJ, Elashoff R, Faries MB and MSLT Group: Final trial report of sentinel-node biopsy versus nodal observation in melanoma. N Engl J Med (2014) 370: 599-609.

2. Morton DL, Hoon DS, Cochran AJ, Turner RR, Essner R, Takeuchi H, Wanek LA, Glass E, Foshag LJ, Hsueh EC, Bilchik AJ, Elashoff $D$ and Elashoff R: Lymphatic mapping and sentinel lymphadenectomy for early-stage melanoma: therapeutic utility and implications of nodal microanatomy and molecular staging for improving the accuracy of detection of nodal micrometastases. Ann Surg (2003) 238: 538-549.

3. Jarrett P, Thomson $\mathrm{W}$ and MacKie RM: Immunohistochemistry and sentinel node biopsy in melanoma: the use of Tyrosinase and melan-A. Br J Dermatol (1999) 141: 361-362.

4. Moroi $Y$ : Significance of sentinel lymph node biopsy in malignant melanoma: overview of international data. Int J Clin Oncol (2009) 14: $485-489$.

5. Al-Hajj M, Wicha MS, Benito-Hernandez A, Morrison SJ and Clarke MF: Prospective identification of tumorigenic breast cancer cells. Proc Natl Acad Sci U S A (2003) 100: 3983-3988.

6. Singh SK, Hawkins C, Clarke ID, Squire JA, Bayani J, Hide T, Henkelman RM, Cusimano MD and Dirks PB: Identification of human brain tumour initiating cells. Nature (2004) 432: 396-401.

7. Kondo $T$, Setoguchi $T$ and Taga $T$ : Persistence of a small sub- 
population of cancer stem-like cells in the C6 glioma cell line. Proc Natl Acad Sci U S A (2004) 101: 781-786.

8. Setoguchi $\mathrm{T}$, Taga $\mathrm{T}$ and Kondo $\mathrm{T}$ : Cancer stem cells persist in many cancer cell lines. Cell Cycle (2004) 3: 414-415.

9. Schatton T, Murphy GF, Frank NY, Yamaura K, Waaga-Gasser AM, Gasser M, Zhan Q, Jordan S, Duncan LM, Weishaupt C, Fuhlbrigge RC, Kupper TS, Sayegh $\mathrm{MH}$ and Frank $\mathrm{MH}$ : Identification of cells initiating human melanomas. Nature (2008) 451: 345-349.

10. Rappa G, Fodstad $O$ and Lorico A: The stem cell-associated antigen CD133 (Prominin-1) is a molecular therapeutic target for metastatic melanoma. Stem Cells (2008) 26: 3008-3017.

11. Grichnik JM, Burch JA, Schulteis RD, Shan S, Liu J, Darrow TL, Vervaert CE and Seigler HF: Melanoma, a tumor based on a mutant stem cell? J Invest Dermatol (2006) 126: 142-153.

12. Boiko AD, Razorenova OV, van de Rijn M, Swetter SM, Johnson DL, Ly DP, Butler PD, Yang GP, Joshua B, Kaplan MJ, Longaker MT and Weissman IL: Human melanoma-initiating cells express neural crest nerve growth factor receptor CD271. Nature (2010) 466: 133-137.

13. Roesch A, Fukunaga-Kalabis M, Schmidt EC, Zabierowski SE, Brafford PA, Vultur A, Basu D, Gimotty P, Vogt T and Herlyn M: A temporarily distinct subpopulation of slow-cycling melanoma cells is required for continuous tumor growth. Cell (2010) 141: 583-594.
14. Kupas V, Weishaupt C, Siepmann D, Kaserer ML, Eickelmann M, Metze D, Luger TA, Beissert S and Loser K: RANK is expressed in metastatic melanoma and highly upregulated on melanoma-initiating cells. J Invest Dermatol (2011) 131: 944-955.

15. Starz H, Siedlecki K and Balda BR: Sentinel lymphonodectomy and s-classification: a successful strategy for better prediction and improvement of outcome of melanoma. Ann Surg Oncol (2004) 11: 162S-168S.

16. Fincher TR, O'Brien JC, McCarty TM, Fisher TL, Preskitt JT, Lieberman ZH, Stephens JF and Kuhn JA: Patterns of drainage and recurrence following sentinel lymph node biopsy for cutaneous melanoma of the head and neck. Arch Otolaryngol Head Neck Surg (2004) 130: 844-848.

17. Shivers SC, Wang X, Li W, Joseph E, Messina J, Glass LF, DeConti R, Cruse CW, Berman C, Fenske NA, Lyman GH and Reintgen DS: Molecular staging of malignant melanoma: correlation with clinical outcome. JAMA (1998) 280: 1410-1415.

18. Yu LL, Flotte TJ, Tanabe KK, Gadd MA, Cosimi AB, Sober AJ, Mihm MC Jr and Duncan LM: Detection of microscopic melanoma metastases in sentinel lymph nodes. Cancer (1999) 86: 617-627.

19. Frank NY, Margaryan A, Huang Y, Schatton T, Waaga-Gasser AM, Gasser M, Sayegh MH, Sadee W and Frank MH: ABCB5mediated doxorubicin transport and chemoresistance in human malignant melanoma. Cancer Res (2005) 65: 4320-4333. 